\title{
Dispersión geoquímica de metales pesados y su impacto en los suelos de la cuenca del río Mantaro, departamento de Junín-Perú
}

\author{
Geochemical dispersion of heavy metals and its impact on the soils of the \\ Mantaro river basin, department of Junín-Peru
}

\author{
Jorge Eduardo Chira Fernández ${ }^{1}$ \\ Recibido: 13/01/2020 - Aprobado: 27/11/2020 - Publicado: 18/06/2021
}

\begin{abstract}
RESUMEN
En esta investigación se evalúa la degradación química y su impacto en los suelos de la cuenca del río Mantaro, en el departamento de Junín- Perú, considerando que esta es producto de procesos, sean de carácter natural como antropogénico.

Los resultados de la investigación permiten determinar áreas que puedan estar impactadas negativamente en lo que se refiere a la distribución de metales pesados en los suelos. El estudio consiste en un muestreo de suelos, habiéndose recolectado 225 muestras superficiales y 64 muestras profundas. La técnica utilizada es especializada, para lo cual se utiliza como instrumento los estándares de calidad ambiental (ECA) de suelos.
\end{abstract}

Al comparar los resultados analíticos con los ECA para suelos de uso agrícola, As (50ppm), Cd (0.9ppm) y Pb (70ppm), estos han sido superados largamente en algunos lugares en As (649ppm), Cd (16.9ppm) y Pb (2021ppm).

La dispersión geoquímica en suelos de plomo, arsénico, mercurio y cadmio está relacionada a la carga metálica de las aguas del río Mantaro que transporta desde el norte, donde se emplaza la minería polimetálica de la Oroya que incluye la presencia de pasivos ambientales mineros.

Palabras clave: Degradación química; metales pesados; análisis multivariado; dispersión geoquímica; ECA suelos.

\begin{abstract}
In this research chemical degradation and its impact on soils of the basin of the Mantaro River in the department of Junin Peru, considering that this is the result of processes are natural and anthropogenic character is evaluated.

Results of the research allow to determine areas that may be negatively impacted regarding the distribution of heavy metals in soils. The study consists of soil sampling, with 225 surface samples and 64 deep samples having been collected. The technique used is specialized, for which the environmental quality standards (EQS) of soils is used as an instrument.

By comparing the analytical results with the EQS for agricultural use soils, As (50ppm), Cd (0.9ppm) and $\mathrm{Pb}(70 \mathrm{ppm})$, these have long been exceeded in some places in As (649ppm), Cd (16.9ppm) and $\mathrm{Pb}$ (2021ppm).

The geochemical dispersion in soils of lead, arsenic, mercury, and cadmium is related to the metallic load of the waters of the Mantaro river that transports from the north, where the polymetallic mining of the Oroya is located that includes the presence of mining environmental liabilities.
\end{abstract}

Keywords: chemical degradation; heavy metals; multivariate analysis; geochemical dispersion; ECA soils.

\footnotetext{
1 Universidad Nacional Mayor de San Marcos, Departamento Académico de Ingeniería Geológica. Lima, Perú.

E-mail: jchiraf@unmsm.edu.pe - ORCID: https://orcid.org/0000-0002-5194-9940
} 


\section{INTRODUCCIÓN}

De acuerdo con el inventario de pasivos ambientales mineros (PAM), el Perú cuenta en la actualidad con 7956, de los cuales 1308 están distribuidos en la cuenca del rio Mantaro (MINEM, 2019) lo cual la convierte en una región prioritaria para establecer mecanismos que permitan tomar medidas dirigidas a remediar o minimizar los efectos en el medio ambiente.

Por otro lado, se cuenta con la Ley General del Ambiente (Ministerio del Ambiente, 2005) y desde ese entonces con normativa ambiental que ha tratado de minimizar el impacto de las actividades antropogénicas, entre ellas la minería.

El impacto en el medio ambiente, que ocasionan los pasivos ambientales mineros y en particular los relaves mineros, es significativo, afectando las fuentes de agua, suelos y atmósfera inclusive; considerando además que los metales pesados se producen naturalmente en el ambiente del suelo a partir de los procesos de degradación de materiales parentales (Wuana \& Okieimen, 2014).

De esta manera, el suelo, sea como producto residual de la degradación física, química y/o biológica o como resultado de la erosión, está sometido a la acción de diversos agentes que pueden llegar a impactar significativamente al punto de deteriorar sus capacidades como base o sostenimiento de la biota (García Guerra, 2009).

Factores tales como el $\mathrm{pH}$, potencial redox y la temperatura, mayormente combinados y frecuentemente inducidos por procesos bacterianos, afectan la solubilidad, movilización y precipitación/deposición de metales potencialmente tóxicos (Siegel, 2002).

Gonzáles V, Valle Sonia, Nirchio M., Olivero J., Tejeda L, Valdelamar J. (2018), identificaron el efecto de la contaminación antrópica en los ecosistemas del estero que proviene posiblemente de las zonas de minería de oro, por una alta contaminación con mercurio.

La presente investigación está referida al impacto que ocasionan los pasivos ambientales mineros en los suelos de la cuenca Mantaro, sector Jauja-Huancayo, contando para ello con la investigación de (Arce \& Calderón, 2018), que identificaron el impacto de los relaves emplazados en La Oroya, en las aguas del rio Mantaro, con énfasis en el plomo. En ese sentido, la hipótesis de la investigación señala que los metales pesados provenientes de los relaves de La Oroya impactan negativamente en los suelos de la cuenca Mantaro, en el sector Jauja-Huancayo (Soto, 2010).

Considerando que la principal fuente que ocasiona el impacto negativo en los suelos entre Jauja y Huancayo, es el Complejo Metalúrgico de la Oroya, situado al norte del área de estudio, (Díaz, 2017), concluye que el mayor contenido de metales pesados se encuentra dentro de 0 a 50 $\mathrm{cm}$ de profundidad y que en otras zonas, la acumulación de contaminantes como plomo y arsénico alcanza promedios muy altos, entre 1000 y $2000 \mathrm{mg} / \mathrm{kg}$, De esta manera, se ha procedido al muestreo y análisis químico de metales pesados en suelos, cuyos resultados son evaluados con los
Estándares de Calidad Ambiental (ECA), establecidos por el Ministerio de Ambiente.

\section{MÉTODOS}

\subsection{Descripción del lugar}

La presente investigación comprende los suelos del valle del Río Mantaro, entre las provincias de Huancayo y Jauja, pertenecientes a la región Junín del centro del Perú). El área de estudio abarca $96 \mathrm{~km}^{2}$, encontrándose a una altitud promedio de 3,300 msnm (Figura 1).

\subsection{Recolección de muestras y preparación}

El muestreo tiene por objetivo investigar la existencia de contaminación del suelo a través de la obtención de muestras representativas con el fin de establecer si el suelo supera o no los Estándares de Calidad Ambiental y/o los valores de fondo establecidos.

La densidad de muestreo básica para suelos superficiales es de una muestra por cada $\mathrm{km}^{2}$. En las zonas urbanas y mineras, la densidad de muestreo es de hasta 2 muestras por $\mathrm{km}^{2}$. En las zonas de topografía o situación complicada, la densidad de muestreo es de 1 a 2 por cada $4 \mathrm{~km}^{2}$. Se siguió el procedimiento establecido por el (MINAM, 2014).

La profundidad de muestreo del suelo superficial es de $0 \mathrm{~cm}$ a $20 \mathrm{~cm}$. Tres a cinco compósitos de muestreo que están dispuestos a $100 \mathrm{~m}$ alrededor de los puntos de muestreo; luego del muestreo compósito se procede al doble cuarteo para obtener la muestra a analizar químicamente, la misma que debe pesar al menos $1000 \mathrm{~g}$.

Los resultados analíticos del muestreo son comparados inicialmente con los estándares de calidad de suelo. Si los valores resultantes superan los valores del ECA y/o los valores de fondo, se determina que el suelo está contaminado. El control de calidad implica la realización de muestreo duplicado, el que representa alrededor de $2 \%$ a $3 \%$ del total.

La densidad de muestreo básica de la muestra de suelo profundo es de una por cada $4 \mathrm{~km}^{2}$. En las zonas de difícil acceso, la densidad de muestreo es de 1 a 2 por cada 16 $\mathrm{km}^{2}$. La profundidad de muestreo es inferior a $150 \mathrm{~cm}$ en zonas llanas, e inferior a $120 \mathrm{~cm}$ en regiones montañosas (Pulido Fernández, 2014).

\subsection{Análisis químicos}

Las muestras de suelo han sido analizadas por 54 analitos: $\mathrm{Ag}, \mathrm{As}, \mathrm{Au}, \mathrm{B}, \mathrm{Ba}, \mathrm{Be}, \mathrm{Bi}, \mathrm{Br}, \mathrm{Cd}, \mathrm{Ce}, \mathrm{Cl}, \mathrm{Co}, \mathrm{Cr}, \mathrm{Cu}, \mathrm{F}, \mathrm{Ga}$, $\mathrm{Ge}, \mathrm{Hg}, \mathrm{I}, \mathrm{La}, \mathrm{Li}, \mathrm{Mn}, \mathrm{Mo}, \mathrm{N}, \mathrm{Nb}, \mathrm{Ni}, \mathrm{P}, \mathrm{Pb}, \mathrm{Rb}, \mathrm{S}, \mathrm{Sb}, \mathrm{Sc}$, $\mathrm{Se}, \mathrm{Sn}, \mathrm{Sr}, \mathrm{Th}, \mathrm{Ti}, \mathrm{Tl}, \mathrm{U}, \mathrm{V}, \mathrm{W}, \mathrm{Y}, \mathrm{Zn}, \mathrm{Zr}, \mathrm{SiO}_{2}, \mathrm{Al}_{2} \mathrm{O}_{3}, \mathrm{Fe}_{2} \mathrm{O}_{3}$ total, $\mathrm{MgO}, \mathrm{CaO}, \mathrm{Na}_{2} \mathrm{O}, \mathrm{K}_{2} \mathrm{O}, \mathrm{C}$ total, $\mathrm{C}$ orgánico, $\mathrm{pH}$.

\subsection{Estándares de calidad ambiental para suelos}

Los resultados analíticos de las muestras de suelo son comparados con los ECA (SINIA, 2017), mostrados en la Tabla 1. Teniendo en cuenta que, de la totalidad de elementos, solamente unos pocos se encuentran normados, la discusión de resultados se abocará al $\mathrm{Pb}, \mathrm{Hg}, \mathrm{As} \mathrm{y} \mathrm{Cd}$. 


\subsection{Análisis estadístico}

Los datos obtenidos son procesados, procediendo a determinar los parámetros estadísticos de cada elemento, en cada nivel de observación.

Se procede a realizar la estadística bivariada y multivariada a fin de establecer las relaciones entre los distintos elementos químicos, tanto a nivel superficial como profundo.

\section{RESULTADOS}

En las Tablas 2 y 3 se aprecian los estadísticos descriptivos de los cinco elementos considerados en el estudio, donde se desprende que los valores medios de arsénico, cadmio y plomo superan los ECA de suelos (uso agrícola), tanto en los niveles superficiales como profundos.

Plomo. Casi el 25\% de las muestras superficiales exceden el ECA (70ppm), llegando a un máximo de

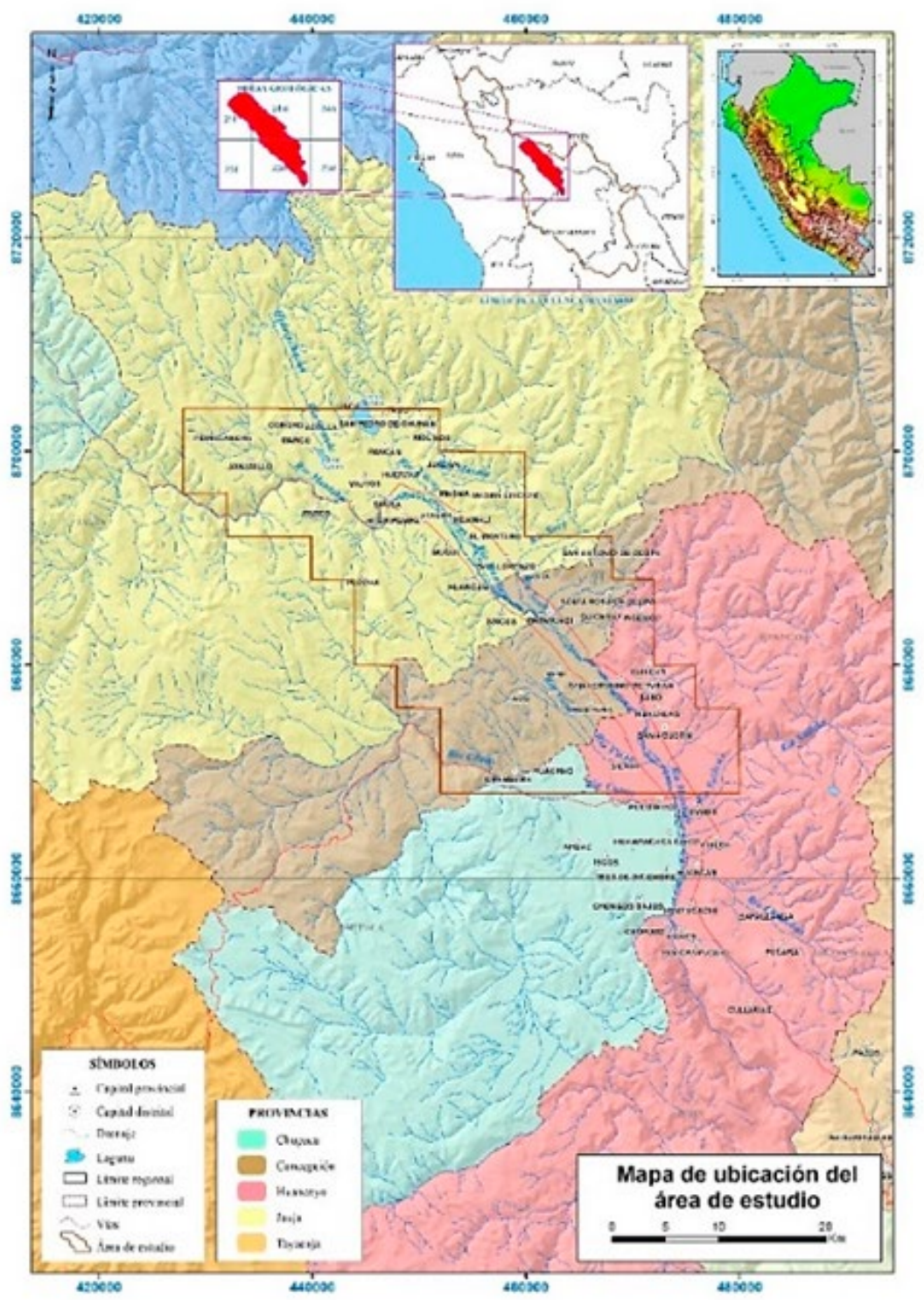

Figura 1. Ubicación de cuenca Mantaro

Tabla 1. Estándares de calidad ambiental (ECAs) para suelo

\begin{tabular}{llccc}
\hline & Parámetro & Suelo agrícola & Suelo residencial/ parques & Suelo comercial/ industrial/ extractivos \\
\hline 1 & Arsénico total $(\mathrm{ppm})$ & 50 & 50 & 140 \\
2 & Cadmio total $(\mathrm{ppm})$ & 1.4 & 10 & 22 \\
3 & Mercurio total $(\mathrm{ppm})$ & 6.6 & 6.6 & 24 \\
4 & Plomo total $(\mathrm{ppm})$ & 70 & 140 & 1200 \\
\hline
\end{tabular}


1439ppm (Figura 2), en tanto que el 11\% de las muestras profundas superan dicho ECA, siendo el máximo de 2021 ppm (Figura 3).

Mercurio. En ninguno de los casos, el mercurio llega a superar el ECA (6.6ppm), aun cuando los mayores valores ocurren en los niveles superficiales (3.44ppm), en tanto que en los profundos no llega a 2ppm. (Figuras 4 y 5 ).

Arsénico. El 26\% de los suelos superficiales exceden el ECA vigente (50ppm), llegando a 437ppm (Figura 6), mientras que en el caso de los suelos profundos el 17\% lo

Tabla 2. Estadísticos descriptivos - Nivel superficial

\begin{tabular}{ccccc}
\hline Elemento & $\mathrm{ECA}(\mathbf{p p m})$ & $\mathbf{N}^{\circ}$ de análisis & Valor máximo & Muestras > ECA \\
\hline $\mathrm{Hg}$ & 6.6 & 225 & 3.44 & 0 \\
$\mathrm{As}$ & 50 & 225 & 437 & 59 \\
$\mathrm{Cd}$ & 0.9 & 225 & 9.54 & 64 \\
$\mathrm{~Pb}$ & 70 & 225 & 1439 & 54 \\
\hline
\end{tabular}

Tabla 3. Estadísticos descriptivos - Nivel profundo

\begin{tabular}{ccccc}
\hline Elemento & $\mathbf{E C A}(\mathbf{p p m})$ & $\mathbf{N}^{\circ}$ de análisis & Valor máximo & Muestras > ECA \\
\hline $\mathrm{Hg}$ & 6.6 & 64 & 1.83 & 0 \\
$\mathrm{As}$ & 50 & 64 & 649 & 11 \\
$\mathrm{Cd}$ & 0.9 & 64 & 16.9 & 12 \\
$\mathrm{~Pb}$ & 70 & 64 & 2021 & 7 \\
\hline
\end{tabular}

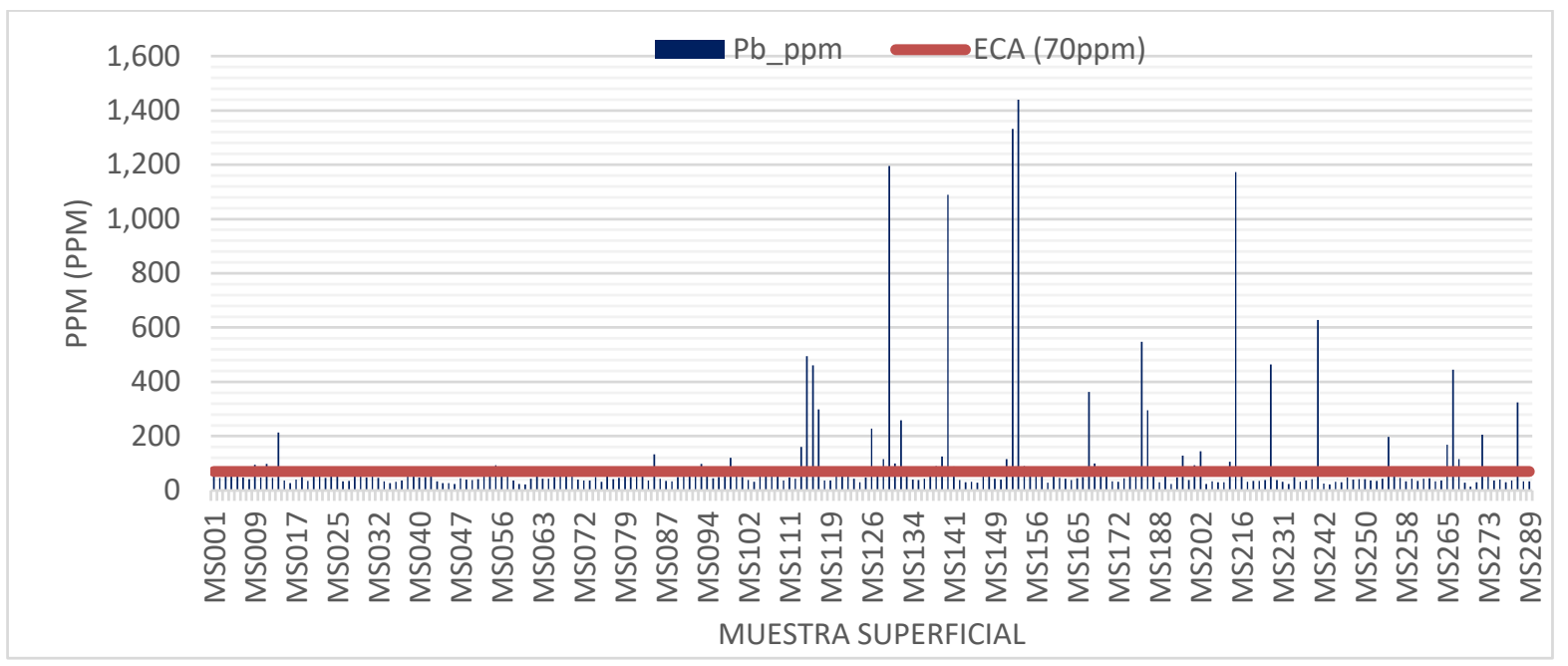

Figura 2. Plomo en suelos superficiales. ECA: 70ppm.

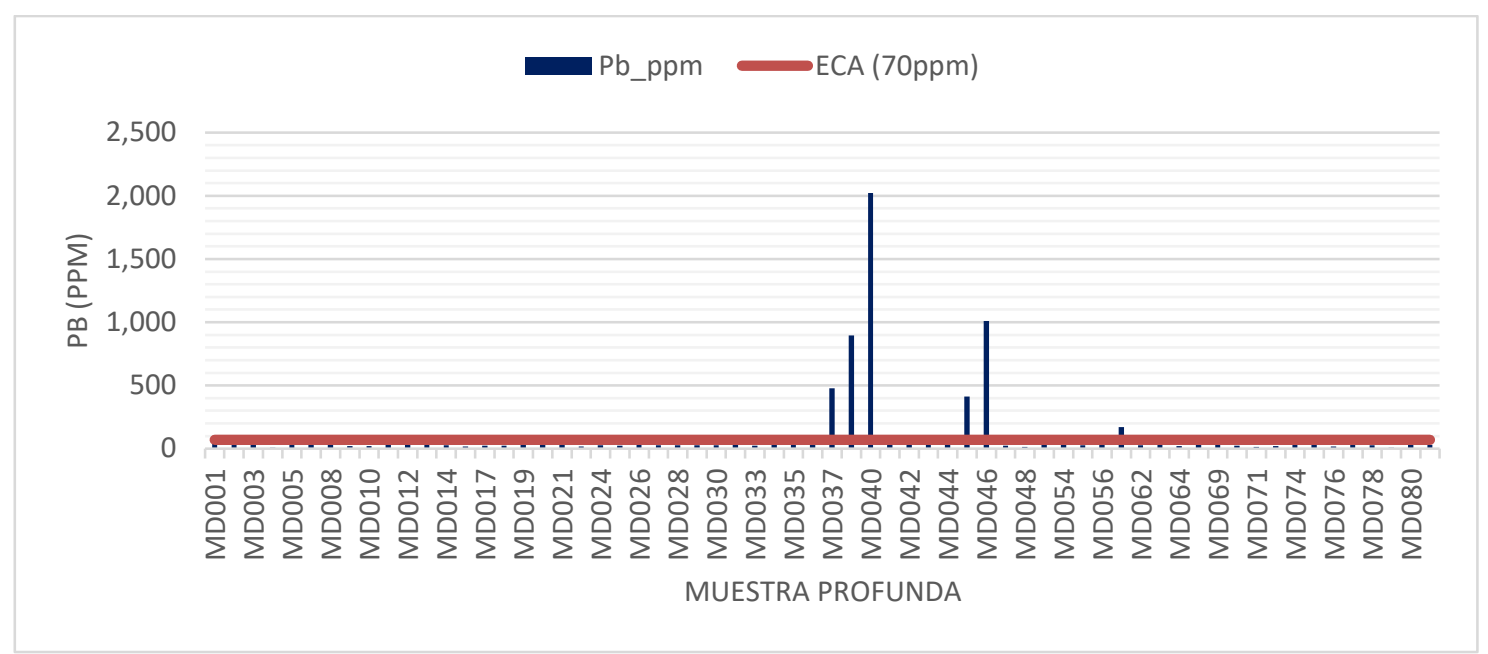

Figura 3. Plomo en suelos profundos. ECA: 70ppm. 


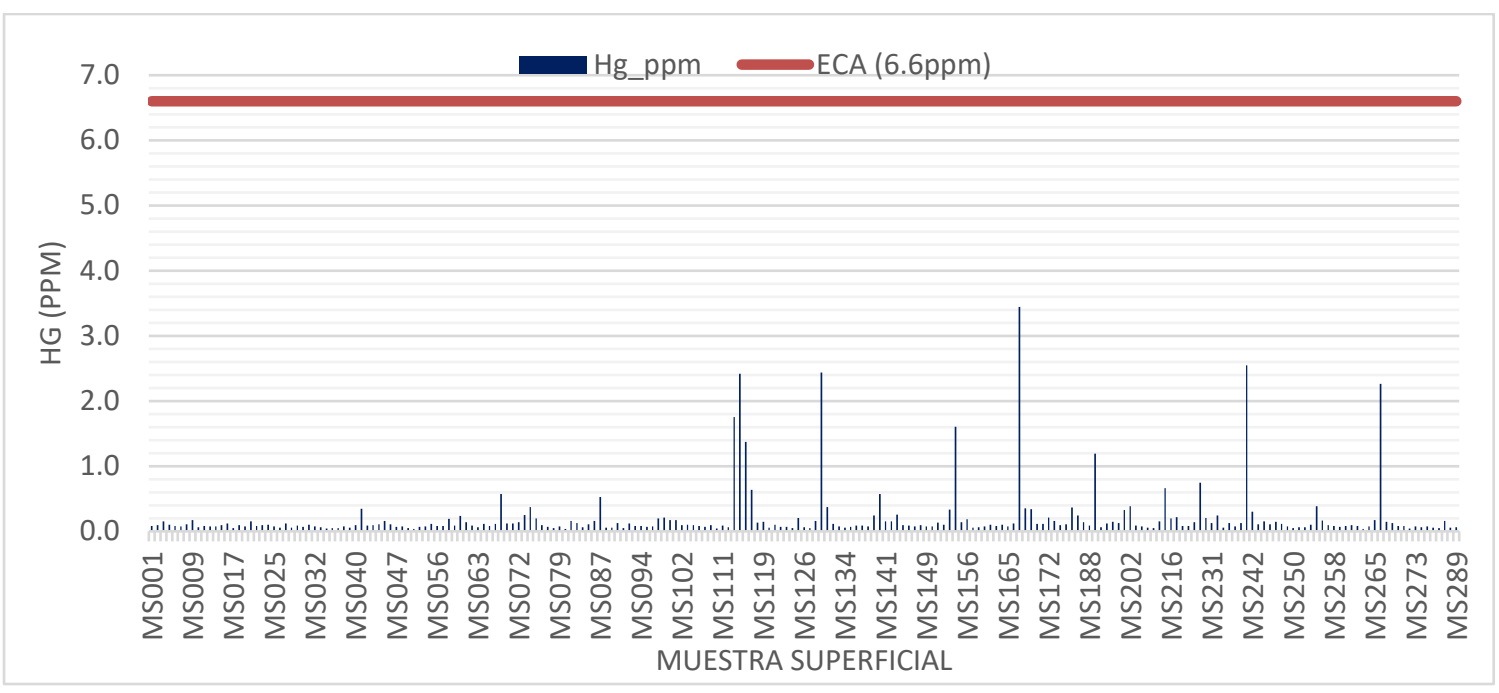

Figura 4. Mercurio en suelos superficiales. ECA: $6.6 \mathrm{ppm}$.

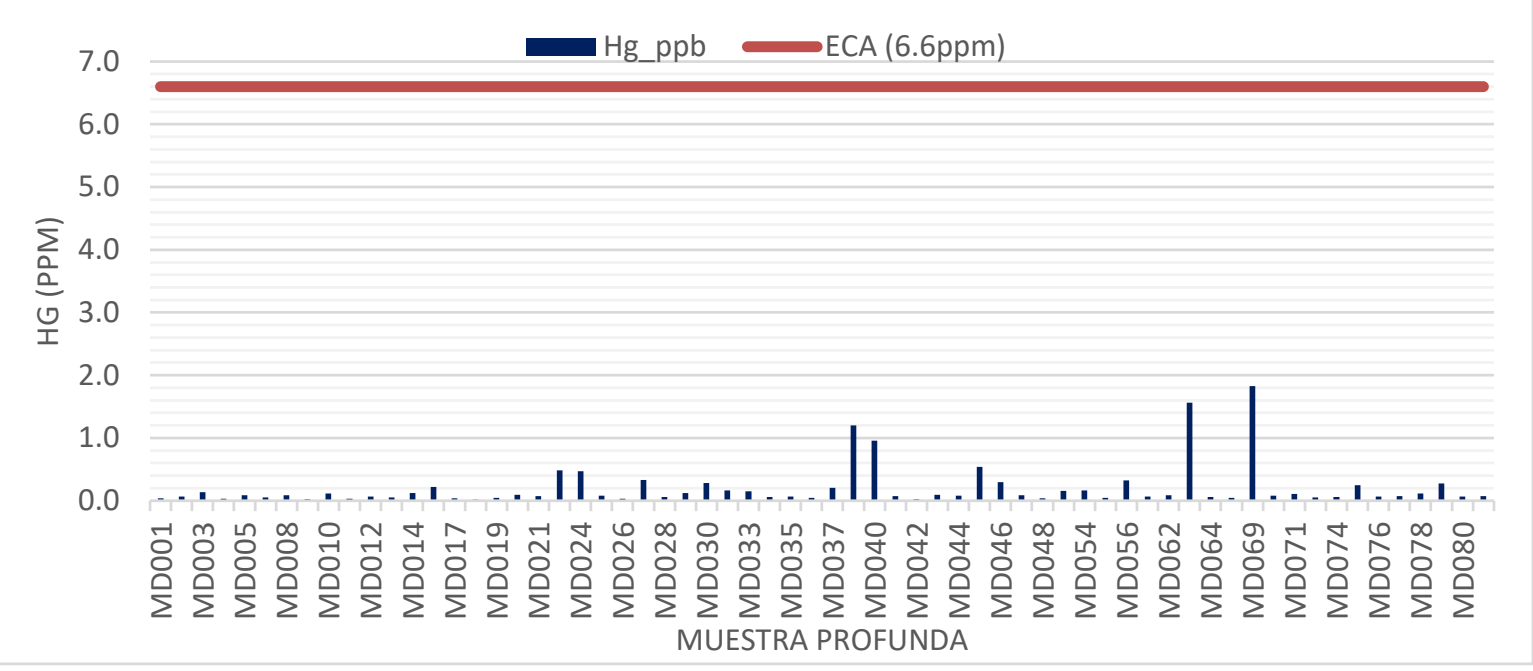

Figura 5. Mercurio en suelos profundos. ECA: $6.6 \mathrm{ppm}$.

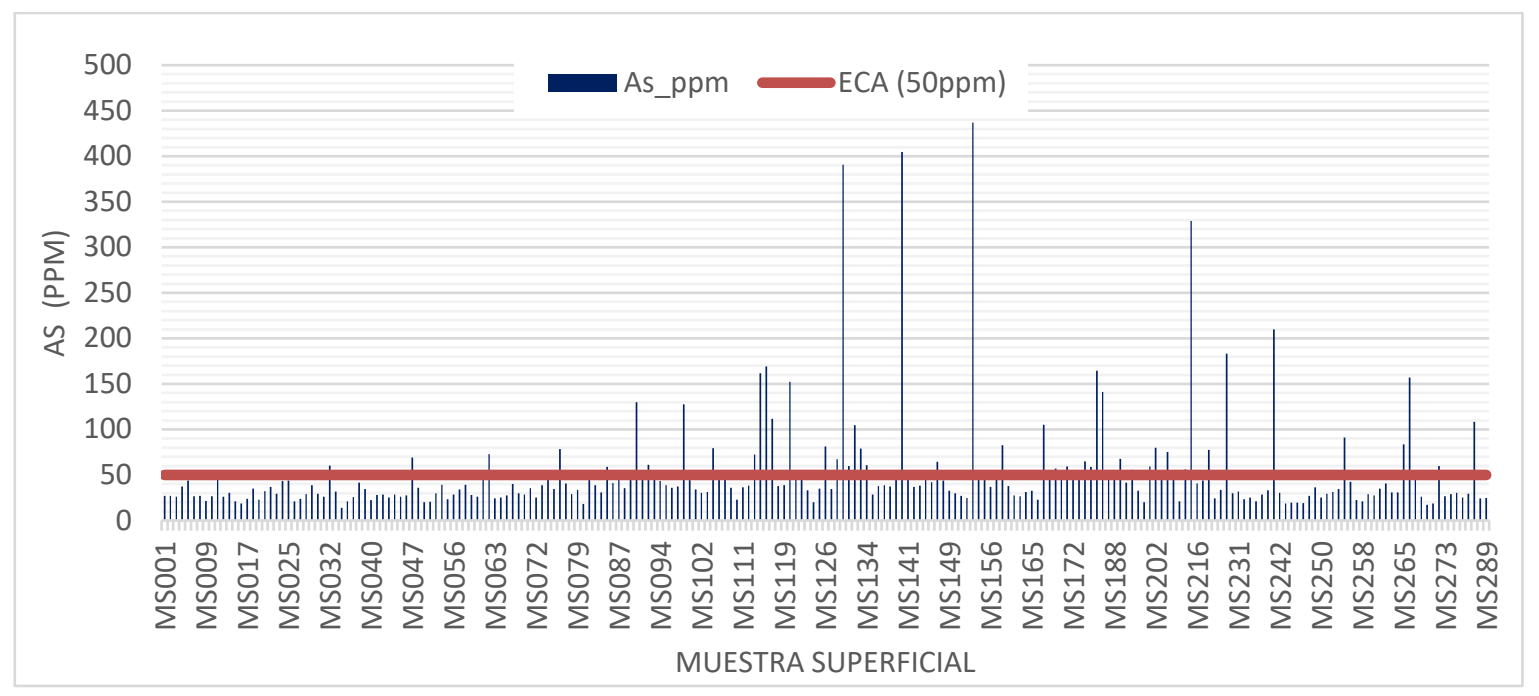

Figura 6. Arsénico en suelos superficiales. ECA: 50ppm. 
supera, siendo el valor máximo de 649ppm, denotando una dispersión más puntual en estos últimos (Figura 7).Cadmio. El 28\% de muestras superficiales superan el ECA vigente $(0.9 \mathrm{ppm})$, mientras que en el caso de las profundas significa el 19\%. Los máximos valores tanto para superficiales como profundas son de 9,54ppm (Figura 8) y 16.9ppm (Figura 9) respectivamente, con un comportamiento similar al plomo y arsénico.

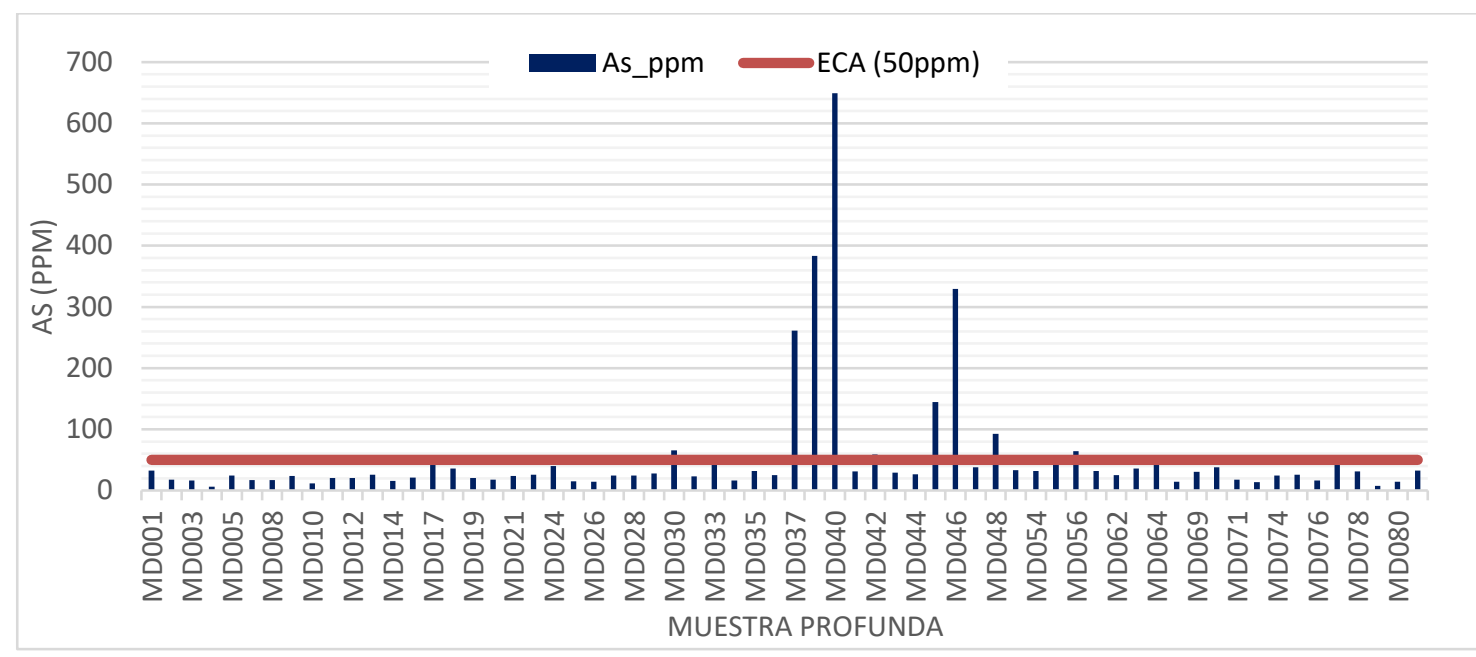

Figura 7. Arsénico en suelos profundos. ECA: 50ppm.

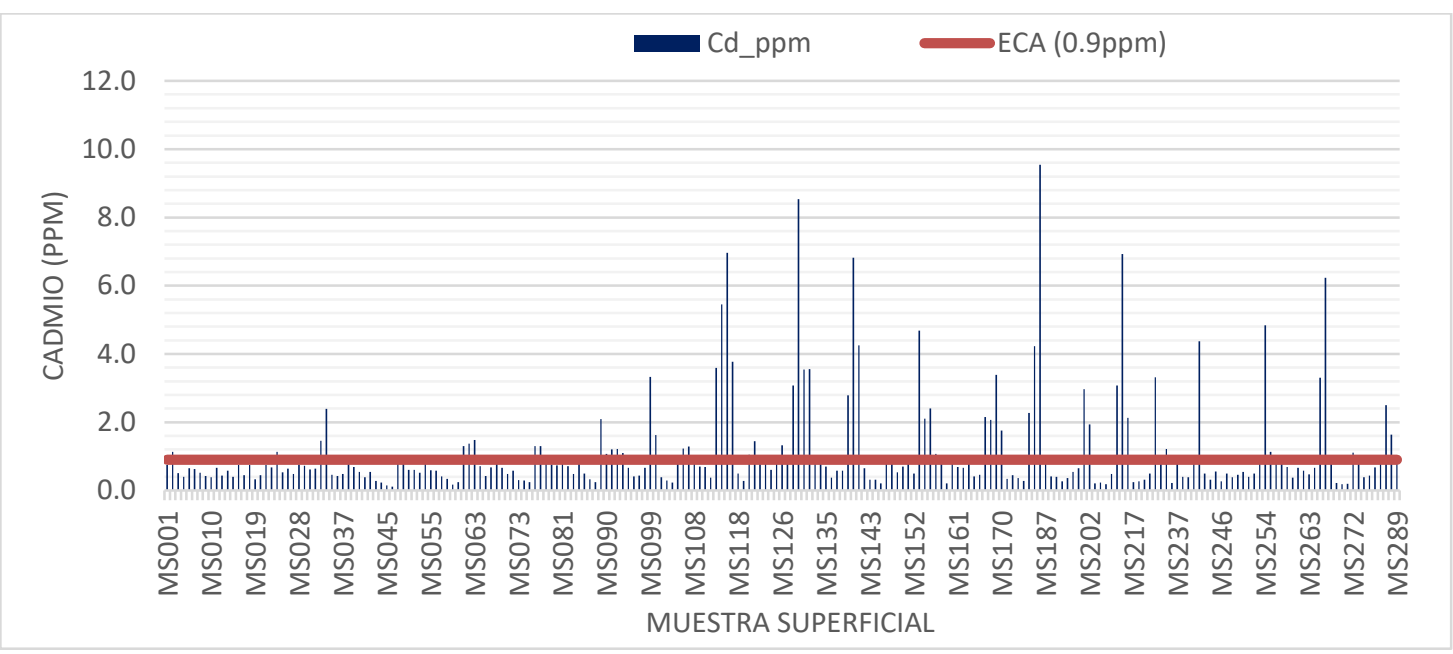

Figura 8. Cadmio en suelos superficiales. ECA: 0.9ppm.

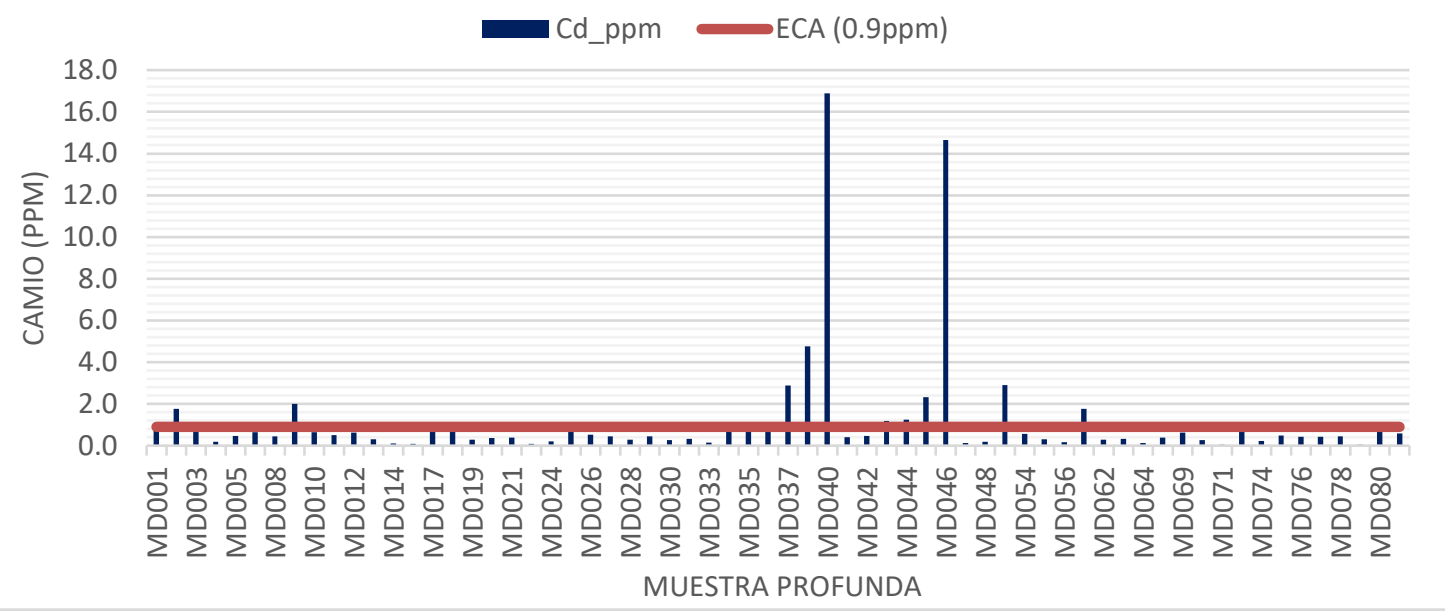

Figura 9. Cadmio en suelos profundos. ECA: 0.9ppm. 


\subsection{Mapas Isovalóricos}

Se han obtenido mapas isovalóricos de los cuatro elementos estudiados, tanto a nivel superficial como profundo, como es el caso de plomo, arsénico, cadmio y mercurio (Figuras $10,11,12$ y 13 respectivamente.

\subsection{Estadística Bivariada}

Los índices muestran una muy fuerte correlación estadística positiva entre el plomo con el arsénico y cadmio. En cuanto al mercurio, muestra correlación fuerte con el plomo y cadmio (Tabla 4)
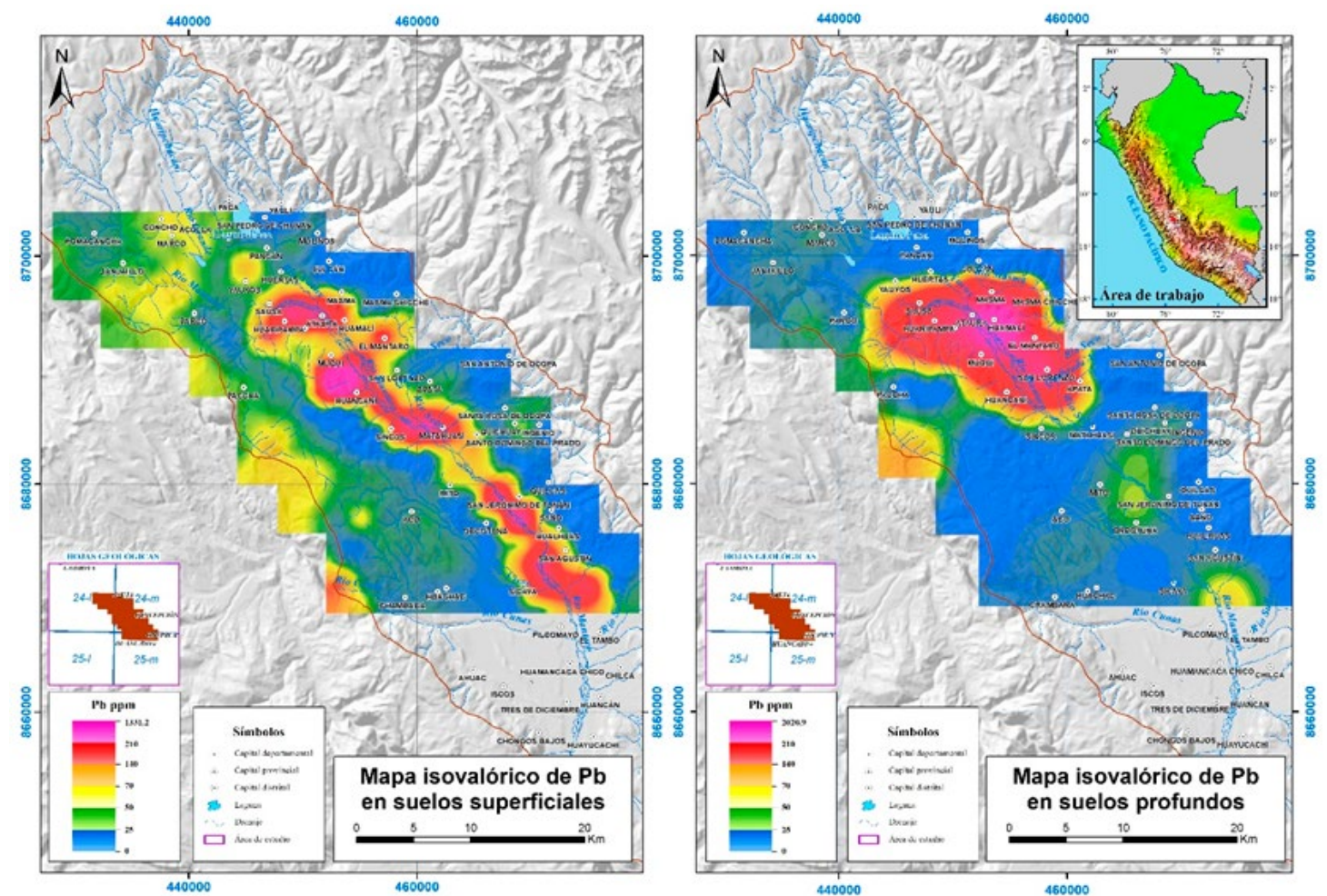

Figura 10. Dispersión del plomo en suelos superficiales y profundos.
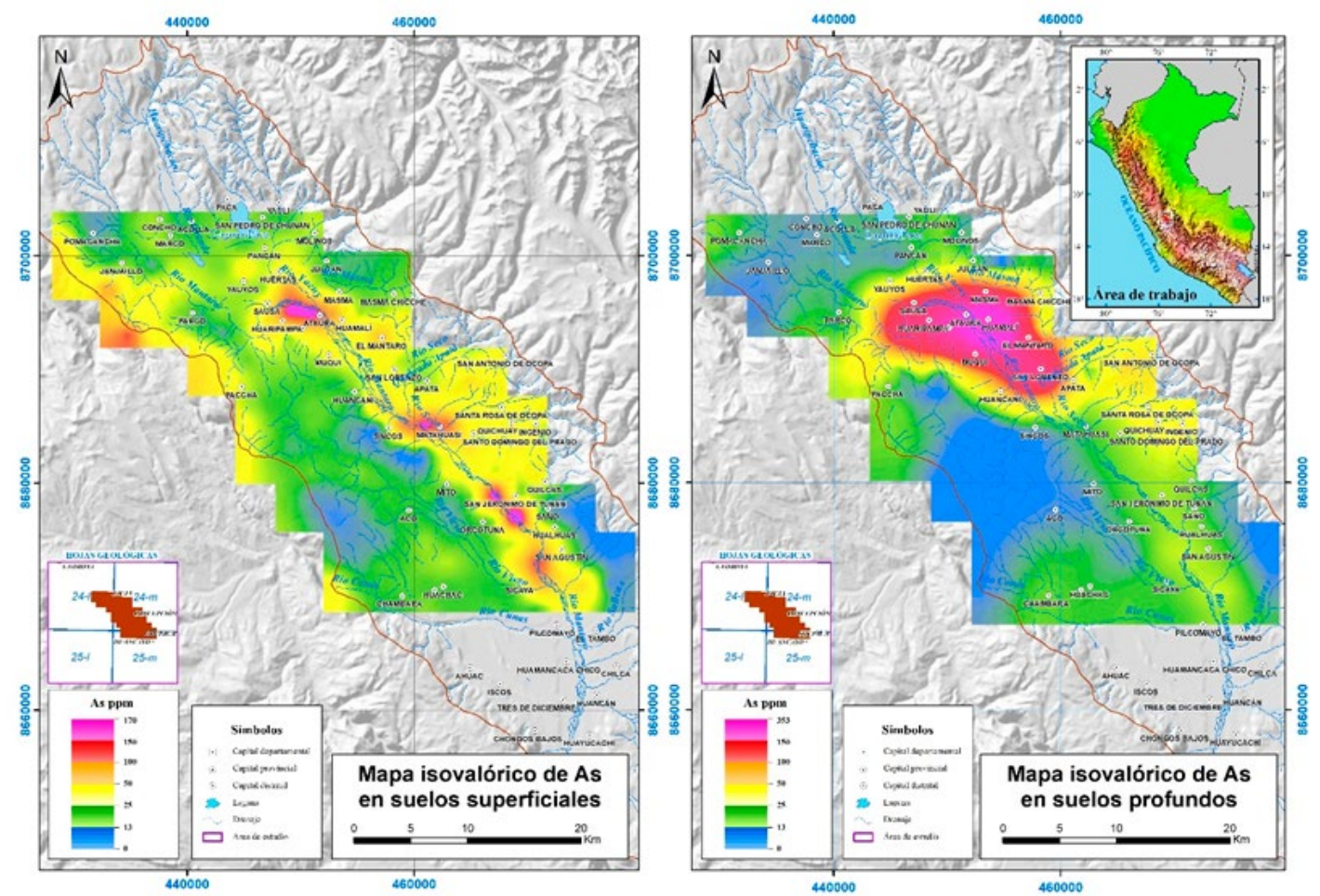

Figura 11. Dispersión del arsénico en suelos superficiales y profundos. 

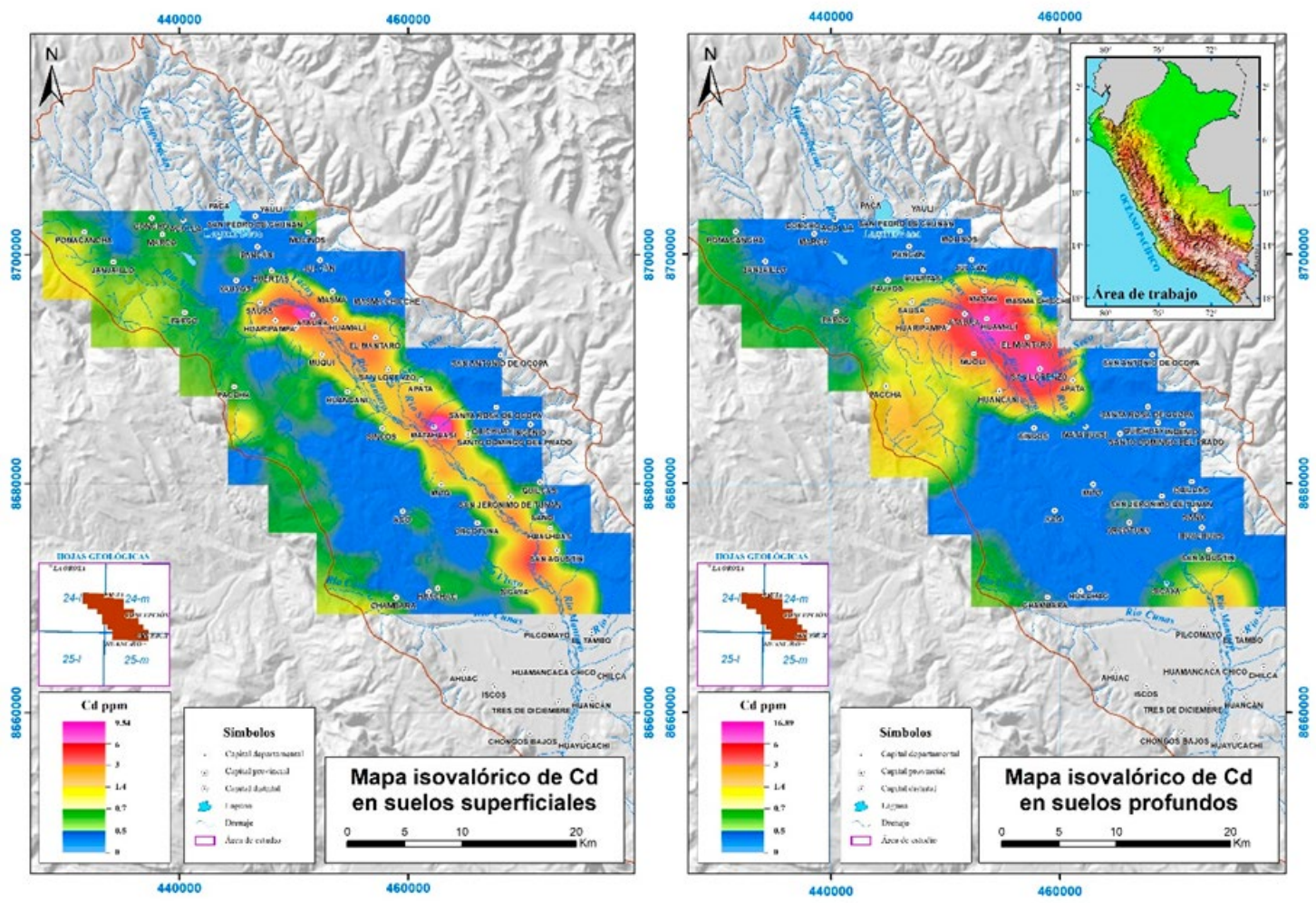

Figura 12. Dispersión del cadmio en suelos superficiales y profundos.
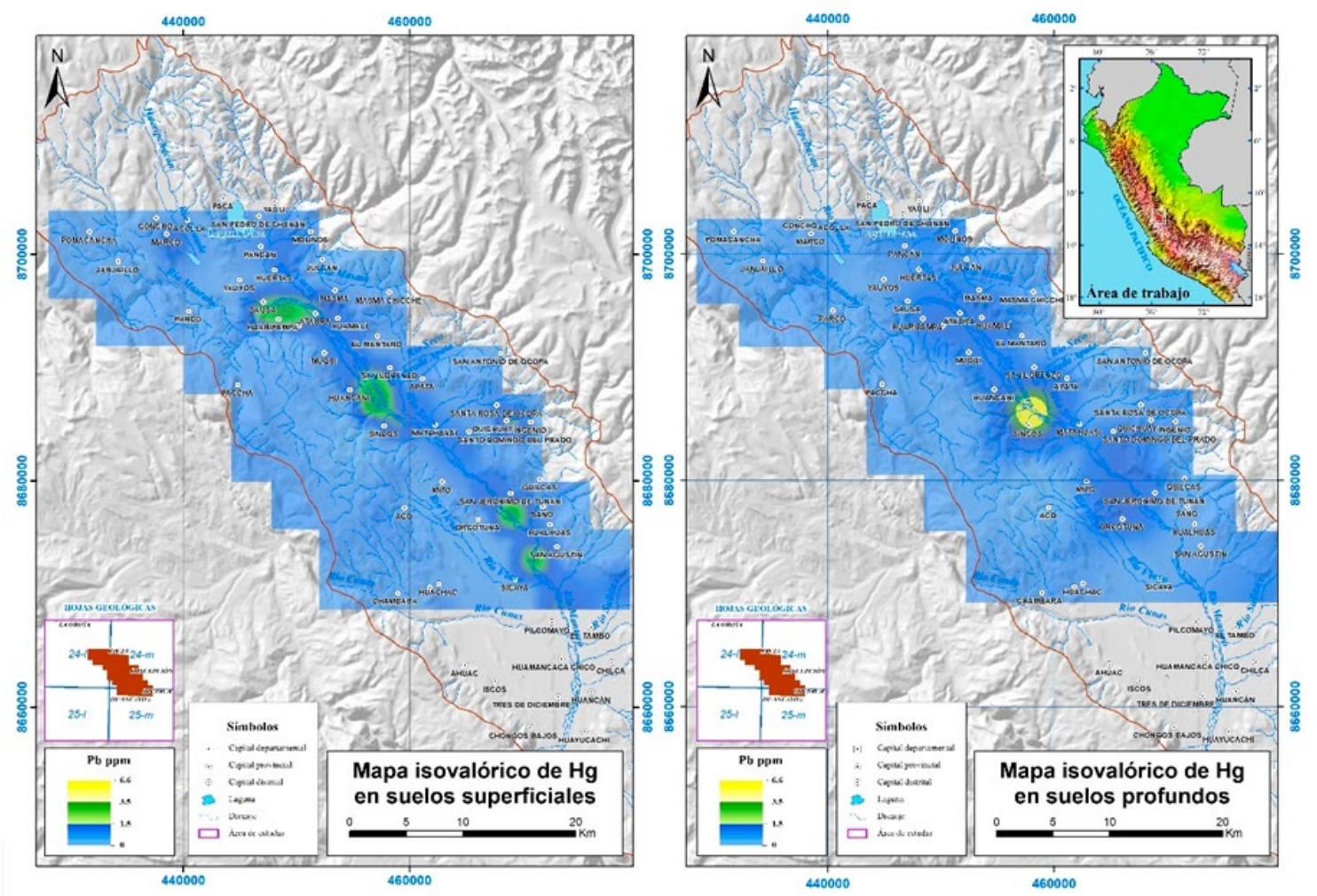

Figura 13. Dispersión del mercurio en suelos superficiales y profundos (Esteban et al., 2007) 
Tabla 4. Correlación bivariada entre algunos elementos metálicos.

\begin{tabular}{ccccc}
\hline & $\mathrm{Hg}$ & $\mathrm{As}$ & $\mathrm{Cd}$ & $\mathrm{Pb}$ \\
\hline $\mathrm{Hg}$ & 1 & 0.496 & 0.507 & 0.504 \\
$\mathrm{As}$ & 0.496 & 1 & $\mathbf{0 . 7 8 5}$ & $\mathbf{0 . 9 1}$ \\
$\mathrm{Cd}$ & 0.507 & 0.785 & 1 & $\mathbf{0 . 7 7 6}$ \\
$\mathrm{Pb}$ & 0.504 & 0.91 & 0.776 & 1 \\
\hline
\end{tabular}

\subsection{Estadística Multivariada}

El análisis de componentes principales permite identificar un segundo componente en el que destaca el plomo, arsénico cadmio y mercurio, que indica la filiación polimetálica de estos y los otros elementos, incluyendo los metales preciosos (Tabla 5).

Por otro lado, los resultados de la prueba de hipótesis para uno de los metales pesados representativos, como es el caso del cadmio, demuestran que existe suficiente evidencia estadística para aceptar que los contenidos de cadmio en el agua se correlacionan con las abundancias de cadmio en los suelos de la cuenca Mantaro.

Nivel de significancia $(\alpha): 5 \%=0.05$.

Estadístico de prueba: Correlación de Pearson.

\begin{tabular}{llcc}
\hline & & Cd (agua) & Cd (suelo) \\
\hline \multirow{3}{*}{ Cd (agua) } & Correlación de Pearson & 1 & $0.605^{* *}$ \\
& Sig. (unilateral) & & 0.000 \\
& $\mathrm{~N}$ & 28 & 28 \\
& Correlación de Pearson & $0.605^{*}$ & 1 \\
Cd (suelo) & Sig. (unilateral) & 0.000 & \\
& $\mathrm{~N}$ & 28 & 28 \\
\hline
\end{tabular}

$\left({ }^{\star *}\right)$ La correlación es significativa en el nivel 0.01 (unilateral). p-valor: 0.000 .

\section{DISCUSIÓN}

Los pasivos ambientales mineros emplazados en la localidad de la Oroya, producto de actividad minera pasada, afectan la calidad el agua del río Mantaro. Los resultados permiten establecer que el agua de la parte superior del valle está cargada de metales y se convierte en uno de los factores principales que afecta los suelos para uso agrícola, más aún si esta es utilizada para irrigar los campos de cultivo del valle.

Como resultado de la evaluación de la calidad de suelos, tres elementos normados superan los ECA de Perú, como son el arsénico, cadmio y plomo, asociación química que se relaciona con la mineralización existente en la localidad de La Oroya, confirmando la proveniencia de dichos metales. En el caso del mercurio, este no supera los referidos ECA.

La mayor concentración de estos metales obedece a la significativa presencia de arcillas, denotándose que el mercurio se fija mayormente en las partes más superficiales debido a que es retenido por la materia orgánica y por los óxidos de hierro y manganeso; en ese sentido, su patrón de dispersión es distinto al de los metales base, los cuales tienen coeficientes de correlación superiores a 0.8 y están más concentrados a profundidad.

Los mapas isovalóricos muestran que el sector más impactado negativamente es entre Sincos (al sur) y Huaripampa, donde se tienen concentraciones de cadmio, arsénico y plomo que superan los ECAs para suelos de uso agrícola, a ambas márgenes del río Mantaro, confirmando el impacto de la actividad minera en el valle.

\section{CONCLUSIONES}

La carga metálica que proviene de La Oroya, lugar donde inclusive se disponen pasivos ambientales mineros,

Tabla 5. Matriz de componentes principales

\begin{tabular}{|c|c|c|c|c|c|c|c|c|c|}
\hline & \multicolumn{9}{|c|}{ Componente } \\
\hline & 1 & 2 & 3 & 4 & 5 & 6 & 7 & 8 & 9 \\
\hline $\mathrm{Cu}$ & -0.039 & 0.983 & -0.07 & -0.023 & 0.032 & -0.011 & -0.042 & 0.038 & 0.058 \\
\hline $\ln$ & -0.11 & 0.963 & -0.065 & 0 & 0.043 & -0.037 & -0.019 & 0.008 & -0.021 \\
\hline $\mathrm{Zn}$ & -0.101 & 0.962 & -0.055 & 0.029 & 0.065 & -0.023 & -0.042 & -0.004 & -0.017 \\
\hline $\mathrm{Sb}$ & -0.115 & 0.953 & -0.042 & -0.001 & 0.021 & -0.067 & 0.005 & -0.012 & 0.114 \\
\hline $\mathrm{Pb}$ & -0.169 & 0.949 & -0.088 & -0.004 & 0.011 & -0.024 & -0.03 & -0.011 & 0.045 \\
\hline As & -0.05 & 0.941 & -0.088 & 0.059 & -0.024 & 0.127 & -0.044 & 0.041 & 0.05 \\
\hline W & -0.013 & 0.848 & 0.024 & 0.009 & 0.076 & -0.078 & -0.062 & -0.008 & 0.263 \\
\hline $\mathrm{Ag}$ & -0.162 & 0.834 & -0.134 & -0.024 & -0.031 & 0.19 & 0.24 & -0.196 & -0.175 \\
\hline $\mathrm{Cd}$ & -0.023 & 0.818 & -0.1 & 0.13 & 0.199 & 0.087 & -0.177 & 0.047 & -0.15 \\
\hline $\mathrm{Au}$ & -0.087 & 0.803 & -0.024 & -0.046 & 0.141 & -0.018 & 0.008 & -0.022 & -0.284 \\
\hline $\mathrm{Mn}$ & -0.103 & 0.666 & -0.162 & 0.22 & -0.023 & 0.204 & -0.101 & 0.429 & -0.119 \\
\hline $\mathrm{Hg}$ & -0.094 & 0.567 & -0.042 & -0.068 & 0.068 & 0.147 & 0.008 & -0.133 & -0.587 \\
\hline $\mathrm{Bi}$ & -0.04 & 0.463 & -0.031 & 0.025 & -0.046 & 0.007 & -0.051 & 0.01 & 0.508 \\
\hline
\end{tabular}

Método de rotación: Varimax con normalización Kaiser. 
ha afectado la calidad química del agua, la cual es utilizada para irrigar los campos de cultivo del sector investigado.

Tres elementos superan largamente los ECA para suelos de uso agrícola, alcanzando valores máximos de 2021 ppm en plomo, 16.9 ppm en cadmio y 649 ppm en arsénico.

\section{AGRADECIMIENTOS}

Un agradecimiento especial a la Alta Dirección del Instituto Geológico Minero y Metalúrgico, así como al equipo de geoquímica, que han colaborado en la generación la información utilizada para la presente investigación.

\section{REFERENCIAS}

Arce, S., \& Calderón, M. (2018). Suelos contaminados con plomo en la Ciudad de La Oroya- Junín y su impacto en las aguas del Río Mantaro. Revista Del Instituto de Investigación de La Facultad de Ingeniería Geológica, Minera, Metalurgica y Geográfica, 20(40), 48-55. https://revistasinvestigacion. unmsm.edu.pe/index.php/iigeo/article/view/14389

Díaz, W. (2017). Estrategia de gestión integrada de suelos contaminados en el Perú. Revista Del Instituto de Investigación de La Facultad de Ingeniería Geológica, Minera, Metalurgica y Geográfica, 19(38), 103-110. https:// revistasinvestigacion.unmsm.edu.pe/index.php/iigeo/ article/view/13575

Esteban, E., Gamarra Gamarra, R., Peñalosa Olivares, J., Moreno, E., Sierra, M., Schmid, T., Carpena Ruiz, R., \& Millán, R. (2007). Rehabilitación de suelos contaminados con mercurio: estrategias aplicables en el área de Almadén. Ecosistemas: Revista Cientifica y Técnica de Ecología y Medio Ambiente, 16(2), 6. https://revistaecosistemas.net/ index.php/ecosistemas/article/view/130

García Guerra, J. A. (2009). Evaluación de la degradación de los suelos naturales de la isla de Tenerife Secuencias edáficas evolutivas y regresivas. https://ialnet.unirioja.es/servlet/ tesis? codigo $=26004$
Gonzáles V, Valle Sonia, Nirchio M., Olivero J., Tejeda L, Valdelamar J, P. F. \& G. K. (2018). Evaluación del riesgo de contaminación por metales pesados $(\mathrm{Hg}$ y $\mathrm{Pb})$ en sedimentos marinos del Estero Huaylá, Puerto Bolívar, Ecuador. Revista Del Instituto de Investigación de La Facultad de Ingeniería Geológica, Minera, Metalurgica y Geográfica, 21(41), 7582. https://revistasinvestigacion.unmsm.edu.pe/index.php/ iigeo/article/view/14995

MINAM. (2014). Guia para el muestreo de suelos (R.M. 085-2014-MINAM) (Vol. 2, p. 91). http://www.minam. gob.pe/wp-content/uploads/2018/07/GUIA-PARA-ELMUESTREO-DE-SUELO.pdf

MINEM. (2019). Inventario de pasivos ambientales mineros. Miniserio de Energia y Minas. http://www.minem.gob.pe/ minem/archivos/ANEXO_RM238.pdf

Ministerio del Ambiente. (2005). Ley General del Ambiente $\mathrm{N}^{\circ}$ 28611. MINAM, Publicada el15 de octubre de 2005, 1-168. https://www.minam.gob.pe/wp-content/uploads/2013/06/ ley-general-del-ambiente.pdf

Pulido Fernández, M. (2014). Indicadores de calidad del suelo en áreas de pastoreo [Universidad de Extremadura, España]. https://dialnet.unirioja.es/servlet/tesis?codigo $=41926$

Siegel, F. R. (2002). Environmental Geochemistry of Potentially Toxic Metals. In Environmental Geochemistry of Potentially Toxic Metals. https://doi.org/10.1007/978-3-662-04739-2

SINIA. (2017). Estándares de Calidad Ambiental para suelo. Ministerio del Ambiente- D.S. N011-2017-MINAM. Decreto Superemo 0011/2017 - Estándares de Calidad Ambiental, 12. https://sinia.minam.gob.pe/normas/ aprueban-estandares-calidad-ambiental-eca-suelo-0

Soto, D. S. (2010). Gestión sustentable del recurso hídrico para la fundación del complejo metalúrgico de La Oroya. http:// cybertesis.uni.edu.pe/handle/uni/612

Wuana, R. A., \& Okieimen, F. E. (2014). Heavy metals in contaminated soils: A review of sources, chemistry, risks, and best available strategies for remediation. In Heavy Metal Contamination of Water and Soil: Analysis, Assessment, and Remediation Strategies (pp. 1-50). https://doi.org/10.1201/ b16566 